\title{
Des gènes de développement de la drosophile codent pour des membres de la superfamille des récepteurs nucléaires
}

Les oncogènes, les gènes de développement et les gènes codant pour différentes protéines intervenant à un niveau ou à un autre de la stimulation hormonale (ou par les facteurs de croissance) sont de même nature. On connaît des oncogènes et des gènes de développement codant pour des analogues des facteurs de croissance (oncogène sis, gènes de développement notch et lin-12 [m/s $n^{\circ} 6$, vol.2, p.340]), de leur récepteur (oncogène $\operatorname{erbB}$, gène de développement sevenless [1]), et pour des facteurs de transcription (oncogène jun, homéogènes, gènes de développement codant pour des protéines à doigts). C'est à ce dernier groupe qu'appartient le gène knirps de la drosophile, dont la mutation aboutit à l'absence de développement de segments abdominaux.

Deux équipes viennent de cloner, en utilisant des stratégies toutes différentes, ce gène et un gène de structure et de localisation voisines. Les chercheurs allemands (laboratoire de H. Jäckele, Münich et Tübingen, RFA) sont partis de la localisation chromosomique de la mutation pour, après microdissection chromosomique et «marche sur le chromosome », isoler un clone codant pour la protéine Knirps[2]. L'autre équipe, de La Jolla en Californie (dirigée par R.M. Evans) a criblé une banque génomique de drosophile à l'aide d'un clone d'ADNc du récepteur de l'acide rétinoïque et a isolé, parmi les clones positifs, une séquence dont la localisation chromosomique correspondait à celle du gène knirps [3].

La séquence en acide aminé déduite de celle des nucléotides ne révélait cependant que $85 \%$ d'homologie avec la protéine Knirps dans les régions $\mathrm{N}$-terminales; le gène a donc locus). Les produits de knirps et de $k n r l$ ont une partie de leurs régions $\mathrm{N}$-terminales qui sont à environ $45 \%$ analogues au domaine $\mathrm{C}$, de liaison à l'ADN, des récepteurs des stéroïdes, des hormones thyroïdiennes et de l'acide rétinoïque.

Les régions plus carboxyterminales, de liaison présomptive d'un ligand, ne ressemblent en rien aux domaines de liaison de l'hormone (ou de l'acide rétinoïque) des autres récepteurs connus. La région conservée de liaison à l'ADN possède, comme pour les autres membres de cette famille, des cystéines en position correcte pour participer à la formation des structures en doigts qui caractérisent divers types de protéines intervenant dans la transcription $(\mathrm{m} / \mathrm{s}$ $n^{\circ} 7$, vol. 3, p. 428) [4]. Le gène knirps est probablement activé par les produits d'un groupe de gènes exprimés dans des cellules du pôle postérieur de l'embryon. On peut supposer que les protéines Knirps et Knrl lient des morphogènes diffusant en un gradient contrôlé par l'activité des gènes «postérieurs » $\left(m / s n^{\circ} 3\right.$, vol. 3, p. 178); le complexe récepteur/morphogène se fixerait alors à des séquences particulières d'ADN, à proximité de gènes dont il modiferait la transcription.

Le récepteur de l'acide rétinoïque est un bon modèle de ce mécanisme, puisque l'acide rétinoïque est un morphogène reconnu qui interviendrait, notamment, dans le développement du bourgeon de l'aile chez le poulet $\left(\mathrm{m} / \mathrm{s} n^{\circ} 3\right.$, vol. 4, p. 196).

A.K.

1. Hafen E, Basler K, Edstroem JE, Rubin GM. Sevenless, a cell-specific homeotic gene of Drosophila encodes a putative transmembrane receptor with a tyrosine kinase domain. Science 1987 ; 236 : 55-63.
2. Nauber U, Pankratz MJ, Kienlin A, Seifert E, Klemm U, Jäckle $H$. Abdominal segmentation of the Drosophila embryo requires a hormone receptor-like protein encoded by the gap gene knirps. Nature $1988 ; 336$ : 489-92. 3. Ovo AE, Ong ES, Margolis JS, Posakony JW, McKeown M, Evans RM. The Drosophila gene knirps-related is a member of the steroid receptor gene superfamily. Nature 1988 ; 336 : 493-6.

4. Helbecque N, Hénichart J.P. Les doigts de zinc, éléments de reconnaissance de l'ADN. médecine/sciences $1988 ; 4: 624-8$.

\section{口 BRÈVES}

L'hypertension artérielle, le cœur et les artères. Asmar et al. [1] ont étudié 16 malades ayant une hypertension artérielle essentielle avant, pendant et après un traitement par un inhibiteur de l'enzyme de conversion de l'angiotensine (IECA), le perindopril. Le produit augmente le débit sanguin de l'avant-bras en accroissant la vélocité et le diamètre artériels (fait remarquable alors que la pression artérielle s'abaisse). La compliance artérielle augmente et la vitesse de propagation de l'onde du pouls diminue (témoignant d'une réduction de la «rigidité » artérielle). Ces modifications sont en partie indépendantes de la baisse tensionnelle et sont probablement dues à l'effet des IECA sur le muscle lisse artériel ; quatre semaines après l'arrêt du traitement, ces modifications artérielles se sont effacées. En revanche, la masse ventriculaire gauche, qui s'est abaissée pendant le traitement, reste encore significativement réduite quatre semaines après l'arrêt.

[1. Asmar RG, et al. Circulation $1988 ; 78: 941-50$.]

$\mathrm{m} / \mathrm{s} n^{\circ} 3$ vol. 5, mars 89 
Les neurones embryonnaires humains peuvent être congelés avant mise en culture ou transplantation dans le cerveau d'un hôte adulte. L'équipe de John Sladek [1], de l'université de Rochester dans l'État de New York (USA), vient en effet de démontrer qu'il est possible de congeler des pièces de tissu nerveux prélevées chez des foetus nés d'IVG entre huit et douze semaines. Les pièces de mésencéphale utilisées dans cette expérience contenaient en particulier l'ébauche du locus niger, c'est-à-dire une population de neurones dopaminergiques. Après congélation dans l'azote liquide, les pièces nerveuses ont été conservées jusqu'à deux mois avant que, après dissociation et réchauffement, les neurones ne soient étudiés. En culture comme dans les transplantations intracérébrales utilisant des singes adultes comme receveurs, les neurones congelés se sont développés normalement et ils ont synthétisé de la dopamine. Cette série d'expériences a deux significations majeures. La première est la possibilité ouverte à la constitution de véritables «banques» de neurones embryonnaires pour la recherche à l'heure actuelle - et pour la thérapeutique, éventuellement. La seconde signification de cette publication, qui n'est pas moins importante, est que les biologistes américains - et la revue Science - ont décidé de braver l'interdiction lancée depuis le mois d'avril dernier par l'administration Reagan contre l'utilisation des tissus embryonnaires humains pour la recherche biomédicale. Cette décision, probablement motivée par des considérations politiques à la veille de l'élection présidentielle américaine, a soulevé un tollé de protestations dans la communauté scientifique américaine. Le débat est d'ailleurs également européen, certains pays de la communauté européenne (dont la RFA) s'engagent dans la même voie que les États-Unis alors que d'autres (dont la France et l'Angleterre) sont favorables à une législation moins restric$\mathrm{m} / \mathrm{s} n^{\circ} 3$ vol. 5 , mars 89 tive tenant compte à la fois des besoins de la recherche biologique et des règles éthiques de nos sociétés [2]. [1. Redmond DE. et al. Science 1988; 242 : 768-71.]

[2. Dickson D. Science 1988; 242 : 1117-8.]

Le développement du cristallin de l'œil dépend d'une production oscillatoire d'un facteur de croissance. Le cristallin est un dérivé ectodermique dont le développement est lié, on le sait depuis longtemps, à la présence dans le voisinage d'une ébauche rétinienne [1]. Le facteur environnemental est donc extrêmement important dans l'ontogenèse de cette structure épithéliale. Brewitt et Clark [2] se sont intéressés de plus près aux mécanismes de cette action de l'environnement et ils se sont en particulier rendu compte que la croissance du cristallin varie considérablement d'un jour à l'autre au cours de l'ontogenèse. Alors que le poids sec d'un cristallin de rat passe en 26 jours de 0,3 à $6,97 \mathrm{mg}$, les oscillations dans la vitesse de croissance varient entre 0 et $87 \%$ d'un jour à l'autre. La vitesse de croissance semble donc suivre une course pulsatile, ce qui a donné l'idée aux auteurs qu'elle suit en fait la libération irrégulière d'un facteur de croissance. En utilisant un système de culture complexe, ils ont effectivement démontré que le cristallin se développe en réponse à l'augmentation de la concentration du PDGF (plateletderived growth factor). Ce facteur est produit par les épithéliums ciliaire et rétino-pigmenté dans l'humeur aqueuse au cours du développement. Sans PDGF, non seulement le cristallin se développe mal, mais encore il apparaît opaque à la fin de la période de développement. Mais que se passe-t-il si l'on cultive un cristallin embryonnaire en présence continuelle de PDGF ? Eh bien, le cristallin atteint effectivement une taille adulte normale, mais il est totale- ment opaque! On ne sait pas encore comment s'opère cette régulation oscillatoire du développement. Cette démonstration introduit cependant une nouvelle dimension temporelle - apparemment importante pour le développement fonctionnel - dans les mécanismes d'action des facteurs qui influencent l'ontogenèse : l'oscillation.

[1. Purves D, Lichtman JW. Sunderland : Sinauer Inc, 1988: 433.]

[2. Brewitt B, Clark JI. Science 1988 ; 242 : 777-9.]

L'endothéline est-elle impliquée dans la pathogénie de l'insuffisance rénale aiguë? L'endothéline est un peptide composé de 21 acides aminés, libéré par les. cellules endothéliales; elle semble agir comme un « modulateur » endogène des canaux calciques sensibles aux dihydropyridines (elle stimule le flux transmembranaire de calcium dans le tissu vasculaire). Dans un rein perfusé isolé, l'endothéline réduit de façon remarquable le débit sanguin et le débit de filtration glomérulaire: l'endothéline est une puissante substance vasoconstrictive ; à une concentration de $200 \mu \mathrm{mol} / \mathrm{l}$, les débits sont diminués de $50 \%$ et annulés à 800 $1000 \mu \mathrm{mol} / \mathrm{l}$; l'effet maximal survient à 30 minutes et dure au moins 90 minutes. Cet effet n'est pas inhibé par le vérapamil, un inhibiteur des canaux calciques. L'angiotensine II exerce une action moins puissante et plus brève: elle débute dès la $8^{\mathrm{e}} \mathrm{minute}$ et dure environ 55 minutes; la perfusion sanguine est diminuée alors que la filtration glomérulaire est peu modifiée; le débit sanguin est réduit de $50 \%$ à une concentration d'e nviron $1000 \mathrm{pmol} / \mathrm{l}$. On peut s'interroger sur le rôle de l'endothéline dans certaines insuffisances rénales aiguës accompagnant les états de choc où le débit sanguin rénal et le débit de filtration glomérulaire sont effon- 
drés. L'hypoxie tissulaire pourrait déclencher la libération d'endothéline dont l'effet persisterait du fait d'une dégradation rénale lente de cette substance. Ces hypothèses demandent à être testées.

[1. Firth JD, et al. Lancet 1988; 2 : 1179-82.]

Un anticorps monoclonal dirigé contre la glycoprotéine plaquettaire $(G p)$ Ilb/IIIa prévient efficacement la récidive d'une thrombose traitée par l'activateur tissulaire du plasminogène (tPA). La glycoprotéine $(\mathrm{Gp})$ IIb/IIIa est une adhésine de la membrane plaquettaire qui reconnaît le facteur von Willebrand, le fibrinogène, la fibronectine et la vitronectine. Cette liaison des plaquettes aux protéines extracellulaires du caillot sanguin et de la paroi vasculaire est indispensable à la formation du thrombus, et les malades dépourvus de ( $\mathrm{Gp}$ ) IIb/IIIa (maladie de Glanzmann) ont un syndrome hémorragique [1, 2]. D'où l'idée que des anticorps dirigés contre cette adhésine pourraient prévenir la formation d'une thrombose vasculaire dans des cas où, de manière aiguë, elle est très redoutée. C'est ce qui se passe en cas de reperméabilisation d'une artère thrombosée lorsque persiste une diminution de calibre (compression, athérome). Sur un modèle animal (le chien), plusieurs équipes américaines de Boston, New York, Burlington (Vermont) et San Francisco viennent de montrer que le fragment $F\left(a b^{\prime}\right)_{2}$ des immunoglobulines (dépourvu du fragment Fc correspondant à la moitié carboxyterminale des chaînes lourdes d'immunoglobuline) d'un anticorps monoclonal anti-(Gp) IIb/IIIa prévenait, dans tous les cas, la re-thrombose d'artères de calibre artificiellement diminué, préalablement reperméabilisées par le tPA [3]. Les autres procédés anti-agrégants ou anticoagulants se sont en revanche révélés soit inefficaces réduite (aspirine). Des tests sur des malades devront maintenant être entrepris afin de déterminer la valeur thérapeutique chez l'homme de ce nouveau procédé [4].

[1. Degos L, Kahn A. médecinel sciences $1987 ; 3: 314-5$.]

[2. Marguerie $\mathrm{G}$, et al. médecinel sciences 1987 ; 3 : 334-42.]

[3. Desnoyer $\mathrm{P}$, et al. médecinel sciences $1988 ; 4$ : 222-30.]

[4. Yasuda $\mathrm{T}$, et al. J Clin Invest 1988 ; 81 : 1284-91.]

Un ARN antisens naturel chez une souris mutante pour le locus shiverer. Il existe deux types de mutation du gène codant pour la protéine basique de la myéline (PBM) chez la souris: la mutation shiverer (shi) comporte une délétion des exons 3 à 7 , entraînant une absence de messager normal. La mutation mld (myelin deficient shimld) associée à une forme plus atténuée de maladie neurologique et permettant l'accumulation d'une certaine quantité de messager fonctionnel, comporte une duplication et un réarrangement du locus. Quelques kilobases en amont d'un gène normal, la souris mld possède un gène dupliqué qui comporte une inversion d'un segment d'ADN contenant les exons 3 à 7 . La séquence de la région d'ADN située à proximité de l'exon 3 inversé révèle de nombreux éléments analogues aux motifs d'ADN qui permettent, au cours de la commutation de classe des immunoglobulines, le réarrangement des segments codant pour les différentes régions constantes $(\mathrm{C} \mu$, $\mathrm{C} \gamma$, etc.) (voir $\mathrm{m} / \mathrm{s}$, suppl. au $n^{\circ} 1$, vol. 5, p. 5) (zones S... du switch). Au cours de ces événements de commutation de classe (dont il existe des équivalents au niveau des gènes du récepteur des lymphocytes $\mathrm{T}$ ), des phénomènes d'inversion et de délétion de fragments sont observés. Il est ainsi possible que des séquences de recombinaison homologue des zones $S$ et situées avant l'exon 3 et après l'exon 7 du gène de la PBM soient responsables de la délétion du gène shi et de l'inversion du gène amont mld. Ce gène mld dupliqué est transcrit en un ARN... partiellement antisens par rapport au messager de la PBM. Quoique l'abondance de ce transcrit anormal soit faible, il se pourrait que sa concentration locale, à proximité du gène normal, fût suffisante pour interférer avec la maturation intranucléaire du prémessager de la PBM, expliquant que la concentration du messager soit réduite chez les souris $\mathrm{mld}$ [1].

[1. Okano $\mathrm{H}$, et al. EMBO J, 1988 ; $7: 3407-12$.]

- Pourquoi les religieuses ne deviennent-elles pas hypertendues? Timio et al. [1] ont comparé dans une étude prospective, sur une période d'environ 20 ans, un groupe de 144 religieuses appartenant à un ordre séculier en Ombrie (Italie) et un groupe de 138 femmes employées vivant autour des monastères : chez celles-ci la pression artérielle, systolique et diastolique, s'élève progressivement avec l'âge (comme cela est habituel dans les pays industrialisés); en revanche, la pression artérielle reste à peu près inchangée pendant la période de 20 ans chez les religieuses (comme cela a été observé dans certaines populations dites primitives). Les deux groupes étaient comparables à l'inclusion; la consommation de $\mathrm{NaCl}$ et d'alcool, ainsi que la prise de poids pendant l'étude ont été identiques dans les deux groupes. La différence persiste quand on compare les religieuses et les employées qui n'ont pas eu d'enfant. Les auteurs suggèrent que le silence, la méditation et l'isolement de la société expliquent cette différence.

[1. Timio N, et al. Hypertension $1988 ; 12$ : 457-61.] 